\title{
EchoGéo
}

$47 \mid 2019$

Nouvelles géographies de la collecte

\section{Les cueillettes commerciales au Québec : capter la diversité socio-environnementale}

Sabrina Doyon

\section{OpenEdition}

Journals

Édition électronique

URL : https://journals.openedition.org/echogeo/16873

DOI : 10.4000/echogeo.16873

ISSN : 1963-1197

Éditeur

Pôle de recherche pour l'organisation et la diffusion de l'information géographique (CNRS UMR 8586)

Référence électronique

Sabrina Doyon, «Les cueillettes commerciales au Québec : capter la diversité socio-

environnementale », EchoGéo [En ligne], 47 | 2019, mis en ligne le 21 avril 2019, consulté le 10 août 2021. URL : http://journals.openedition.org/echogeo/16873 ; DOI : https://doi.org/10.4000/echogeo. 16873

Ce document a été généré automatiquement le 10 août 2021.

EchoGéo est mis à disposition selon les termes de la licence Creative Commons Attribution - Pas d'Utilisation Commerciale - Pas de Modification 4.0 International (CC BY-NC-ND) 


\title{
Les cueillettes commerciales au Québec : capter la diversité socio- environnementale
}

\author{
Sabrina Doyon
}

\section{Introduction}

1 Au Québec, les activités de cueillettes commerciales de produits issus de la cueillette de plantes, de rameaux de branches, de racines d'arbres, de bourgeons, de petits fruits et de champignons se déroulent sur l'ensemble du territoire, de la taïga, aux massifs feuillus du sud de la province en passant par les forêts boréales (illustration 1). Les produits de ces cueillettes sont nommés par l'administration québécoise et canadienne des " produits forestiers non ligneux" (PFNL), et comprennent tous les produits d'origine biologique, autre que le bois et la faune, possédant une valeur économique provenant des forêts ${ }^{1}$.

2 Les cueillettes s'effectuent dans trois types de tenures qui, au Québec, sont réparties selon un gradient latitudinal : premièrement, des territoires publics sauvages situés au nord de la limite d'attribution de droits de coupe forestière, deuxièmement, des territoires publics où la vocation principale, contemporaine et historique, est l'extraction industrielle du bois et, enfin, des terres privées souvent aux marges de l'écoumène agricole, comme des lots de forêts, généralement situés au sud de la province (illustration 2).

3 Les gouvernements provincial et fédéral affirment être intéressés par ces produits, mais leurs investissements, tant en recherche et développement qu'en accompagnements ou en appuis à la mise en marché, demeurent marginaux. Certains gouvernements municipaux régionaux éloignés des grands centres urbains revendiquent quant à eux des financements publics pour structurer ce marché afin de venir en aide à leur économie locale. Les PFNL leur apparaissent prometteurs pour favoriser des opportunités de développement régional. Ils sont promus, d'une part, 
comme une façon de mieux mettre en valeur la diversité des ressources de la forêt, des espaces forestiers en génération suite aux coupes et des milliers d'hectares de parcelles agricoles dévalorisées ou en friche, et, d'autre part, pour contribuer à l'économie régionale, à l'occupation du territoire par ses habitants et ses travailleurs, à la création d'organisations socio-économiques locales structurantes pour gérer ces activités (telles des coopératives) et à la création d'emplois.

Illustration 1 - Domaines bioclimatiques du Québec

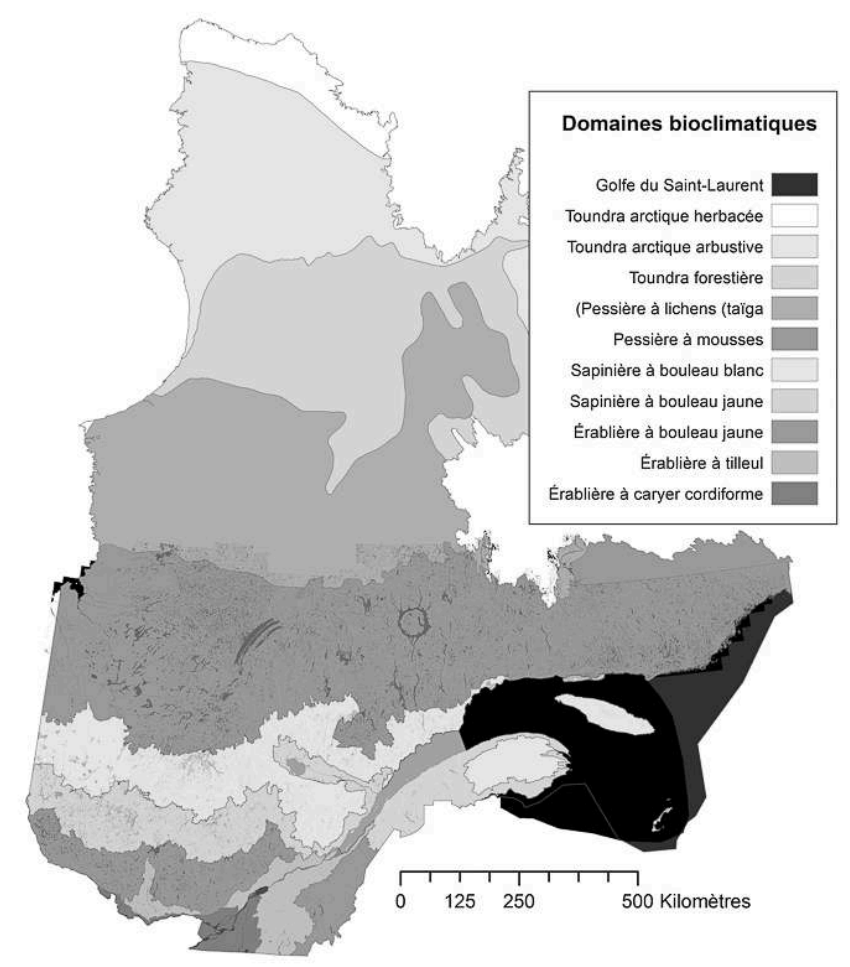

Auteur : Marc-André Brochu.

La valeur annuelle de certains produits forestiers non ligneux a été estimée à $283 \mathrm{M} \$$ pour l'ensemble du Québec en 2006 (MRNF, 2009). Cette valeur inclut la valeur de vente de la production de sirop d'érable (164 M\$), les bleuets sauvages (68 M\$) et les arbres de Noël$^{2}(51 \mathrm{M} \$)$. En considérant les différents PFNL exclus de cette estimation, nous supposons que la valeur de tous les PFNL du Québec représente une valeur annuelle d'au moins 300 M\$ (MRNF, 2003). À l'échelle de tout le Canada, les produits de ces cueillettes généreraient 1,26 milliard de dollars de ventes annuelles (Mitchell et al., 2010). Ces activités demeurent cependant marginales puisqu'elles représentent moins de $5 \%$ de la valeur de vente de bois (pâtes et papiers, bois d'œuvre et granules pour combustion) généré annuellement par l'industrie forestière canadienne. Murphy et al. (2017, p. 191) soulignent d'ailleurs que les PFNL sont considérés comme « mineurs » par les différents paliers de gouvernement parce qu'ils sont saisonniers, localisés surtout dans «l'arrière-pays » et participant plus généralement à une économie informelle, voire souterraine.

Dans cet article nous souhaitons contribuer à la compréhension des ressorts de ces pratiques de cueillette et éclairer les défis propres à cette activité aux caractéristiques diversifiées. Depuis le début des années 1990, les potentialités de commercialisation des 
PFNL du Québec ont fait l'objet d'études, principalement issues du domaine de la foresterie, exposant les revenus intéressants que ces ressources pourraient offrir selon les différentes gammes de produits frais ou transformés et insistant sur l'éventail de produits encore non commercialisés qui pourraient permettre le développement de nouveaux marchés selon des demandes internationales (Fugère et Léveillé, 2005; Gélinas et al., 2016 ; Turgeon, 2003 ; Biopterre, 2015, 2016 ; Brodeur et al., 2012 ; Charron et al., 2014). Ce portrait n'éclaire pas cependant les pratiques qui doivent être mises en œuvre par les cueilleurs qui sont au cœur du succès de ces initiatives. L'examen de ces activités permet de révéler la pluralité des types de cueillettes et d'aborder les enjeux auxquels les cueilleurs font face. Comment la cueillette d'espèces sauvages s'inscrit-elle dans un processus où les cueilleurs sont amenés à déployer un savoir, des ressources et des organisations différentiés dans une diversité de lieux ?

Il est intéressant d'éclairer cette situation à la lumière de la perspective «d'accumulation par captation » proposée par Tsing $(2015$, p. 63), soit la façon dont le vivant est coopté pour favoriser une accumulation sans qu'il y ait eu de valeur investie dans sa production ou dans les conditions dans lesquelles il est extrait. Tsing nous invite à observer comment cette forme d'accumulation prolifère sur «les ruines du capitalisme » $(2012,2015)$, tirant avantage des qualités et des temporalités mêmes de la nature. Dans le cas des divers types de cueillettes au Québec, cette perspective permet d'observer comment certains sites, abandonnés après avoir été vidés de leurs matières premières ou considérés comme improductifs (en raison de leur faible densité ligneuse de qualité ou de la pauvreté des sols), des avatars de ces « ruines du capitalisme », sont investis par des acteurs et des processus qui évoluent à la fois dans et pour l'accumulation capitaliste, mais qui en sont également à la marge par l'organisation de leur travail et les ressources qu'ils puisent et mobilisent, évoluant dans un espace "péricapitaliste» (Tsing, 2015, p. 63). Comment des activités et des ressources se trouvant à la périphérie du capitalisme contribuent-elles à la valeur des PFNL et au système capitaliste dans lequel elles se déploient? L'analyse des lieux et des pratiques de cueillette de PFNL peut-elle éclairer ce qu'implique la rencontre d'activités capitalistes et non capitalistes?

7 Les données d'une recherche qualitative ont permis de répondre à ces questions ${ }^{3}$. La stratégie de recherche a permis la réalisation d'une recension des écrits sur la question des produits forestiers non ligneux au Québec ainsi que des entretiens avec des acteurs québécois du milieu et des cueilleurs. Les participants ont été identifiés lors d'une recension de plus de 250 initiatives comportant des utilisations et des mises en valeur de la nature qui favorisent la conservation environnementale et qui sont alternatives aux modes de production conventionnelle, quinze cas impliquant la cueillette dans le Bas-Saint-Laurent ont fait l'objet d'entrevues semi-dirigées et d'une ethnographie de leurs caractéristiques, notamment leur position face au système économique global et local ainsi que les traits de leurs rapports à la nature, à la conservation environnementale et à leur région. Des analyses thématiques des entretiens et des documents ont été ensuite menées. 


\section{De l'exclusion à l'ouverture : émergence des cueillettes commerciales}

8 Dès la fondation de la Nouvelle-France, la cueillette a été au cœur de l'économie de subsistance et des rapports sociaux des habitants, autochtones ${ }^{4}$ et allochtones, offrant un apport de produits alimentaires et médicinaux de base (Fortin et Lechasseur, 1993). Si la cueillette de subsistance s'est maintenue à très fine échelle jusqu'à nos jours, la cueillette commerciale a été reléguée à un rôle quasi inexistant jusque dans les années 1980. La structuration des établissements humains et de l'économie paysanne, le développement industriel, l'exode rural, ainsi que la dégradation et l'éloignement graduel des habitats propices à la cueillette dans l'écoumène agricole et la privatisation de la terre ont notamment contribué à cette situation.

L'absence de cueillettes commerciales est aussi étroitement liée à l'interdiction, pendant plus d'un siècle, pour l'ensemble de la population québécoise, d'accès aux immenses forêts publiques. En effet, le régime forestier du Québec a, dès le début du $19^{\mathrm{e}}$ siècle concédé l'exploitation et la transformation des forêts à des compagnies privées, propriétés de riches familles, aussi nommées les «Barons du bois». Les terres publiques, alors nommées terres de la couronne, furent concédées pour des termes de 99 ans à ces compagnies, qui obtinrent l'usage exclusif du bois sur ces territoires. En prétextant le risque d'incendie que des fréquentations incontrôlées pouvaient occasionner, des barrières interdirent l'accès aux autres ressources, particulièrement les poissons et les gibiers (Abanda et al., 2016 ; Gagnon, 2009; Lapointe, 2010). Des parties de concessions avaient ainsi pu être organisées en clubs privés de chasse et de pêche. Cette situation a nourri une insatisfaction croissante au sein de la population québécoise sur fond d'enjeux d'autonomie et de souveraineté. En 1977, un an après l'accession au pouvoir du Parti Québécois, l'ouverture subite des barrières des concessions fut ordonnée par le gouvernement provincial ${ }^{5}$. Cette ouverture a permis le libre accès pour la population du Québec aux forêts et aux milliers de kilomètres de pistes héritées de décennies d'extraction du bois. Dès lors, les activités de cueillettes commerciales ont pu se développer et se structurer, notamment dans les espaces de coupe à blanc et de brûlis, des espaces abandonnés par l'industrie forestière.

Malgré ces contraintes d'accès, la richesse de la flore et des champignons présents au Québec avait graduellement été révélée par quelques scientifiques, de la fin du XIX ${ }^{e}$ siècle aux années 1960. Puis, des percées de vulgarisation ont marqué les années 1970 et 1980 permettant l'émergence de nombreux groupes d'adeptes de loisirs scientifiques naturalistes. La démocratisation et la diffusion progressive des savoirs cumulés en matière de plantes et de champignons comestibles ont pris un véritable essor durant les années 1990, inspirant encore aujourd'hui les jeunes enthousiastes qui se lancent dans l'aventure de la cueillette et de la transformation de plantes et de champignons sauvages. Les savoirs floristiques et mycologiques ont longtemps évolué parallèlement ; ils ont convergé pour une poignée de cueilleurs qui ont commencé les premiers partenariats avec la gastronomie fine à la fin des années 1980. Parallèlement, l'herboristerie et plusieurs voies de médecines alternatives se sont consolidées permettant l'émergence d'entreprises importantes au Québec.

11 Les activités de cueillettes sont engagées dans un processus de structuration et de professionnalisation depuis un peu plus d'une décennie. Le premier colloque sur les PFNL organisé à La Pocatière s'est tenu en 2004, mettant en contact des coopératives de 
travailleurs forestiers, des cueilleurs et des transformateurs ainsi que quelques acteurs régionaux de développement. L'année suivante, l'Association pour la commercialisation des champignons forestiers est fondée (elle deviendra l'Association pour la commercialisation des PFNL en 2012). Aussi, des « portraits régionaux » ont été rédigés décrivant leurs PFNL et leurs possibilités de valorisation économique, notamment au Saguenay-Lac-Saint-Jean, au Kamouraska, en Mauricie, en Abitibi et en Outaouais. Des collaborations avec des organisations aptes à l'acquisition et au transfert de connaissances apparaissent et des collaborations dans le cadre de projets régionaux comme l'appellation «Agroboréal» au Saguenay-Lac-Saint-Jean prennent forme et montrent un désir de s'approprier et de mettre en valeur ces ressources et leurs territoires. La structuration de la cueillette est maintenant renforcée par des formations techniques collégiales offertes au Lac-Saint-Jean, en plus de cours privés pour former des cueilleurs et des transformateurs de PFNL, notamment des cuisiniers, dans les régions de la Mauricie, de Lanaudière et des Laurentides. Ces initiatives témoignent d'un intérêt régional pour la structuration et le développement de ces activités, qui permettraient notamment de favoriser l'économie locale, l'emploi, le maintien des jeunes en région, la diversification économique et l'occupation du territoire. Des travaux en foresterie le suggèrent avec le cas des « forêts de proximité et communautaires " (Bouthillier et al., 2011), où se déploient des activités économiques diverses et d'envergure différentiées afin de favoriser le développement régional. Les problèmes économiques des régions du Québec contribuent à la dévitalisation sociale et à la perte de pouvoirs politiques, et la territorialisation des activités, le maintien des emplois, la diversité économique sont des aspects importants auxquels peuvent contribuer les PFNL et qui s'inscrivent dans les préoccupations du développement régional ${ }^{6}$. Ces initiatives se comptent cependant encore sur les doigts de la main et sont marginales dans le portrait plus général de la cueillette, qui, comme nous le verrons, s'inscrit dans une logique de production de valeur et d'accumulation capitaliste où les bénéfices régionaux, sociaux, politiques et économiques, demeurent accessoires.

\section{Les cueilleurs et leur territoire}

12 Au Québec, la majorité du volume des cueillettes provient de terres publiques (elles constituent 91,6\% du territoire, soit $1372021 \mathrm{~km}^{2}$ ). Toutes tenures confondues, les écosystèmes forestiers et associés où peuvent se déployer des cueillettes s'étendent sur plus de la moitié du territoire continental, soit $761100 \mathrm{~km}^{2}$ (voir illustration 2). De ce territoire forestier, $451331 \mathrm{~km}^{2}$ ont pour vocation principale l'aménagement forestier industriel extensif dont $336586 \mathrm{~km}^{2}$ sont accessibles et hors aires protégées et sont les lieux où se déroulent principalement les cueillettes. De cette superficie, $20 \%$ sont en tenure privée, principalement localisée au sud de la province, dans la vallée du fleuve Saint-Laurent (Forestier en chef, 2018). Les cueillettes s'effectuent dans des espaces variés et concernent une diversité de plantes : plus particulièrement, on retrouve des plantes de milieux ouverts ${ }^{7}$, de milieux humides et rivulaires ${ }^{8}$ et de sous-bois ${ }^{9}$. Il y a aussi les branchages ligneux ${ }^{10}$, les sèves ${ }^{11}$ et les champignons ${ }^{12}$. Ces produits peuvent être utilisés frais ou transformés, notamment dans une chaine de production industrielle pour la production d'huile essentielle et de produits pharmaceutiques, ou de façon plus artisanale, pour la production de tisanes, de sels, de poudres, de décoctions, de crèmes, etc. 
En terres publiques, l'immense majorité des espèces de plantes et de champignons peut être cueillie sans limites quant aux quantités prélevées. Hormis le bois des essences d'arbres reconnues commerciales, comme l'épinette noire, le sapin, la pruche, l'érable, le bouleau jaune ${ }^{13}$, c'est une grande part des ressources biologiques renouvelables présentes sur d'immenses territoires appartenant au bien commun auquel les cueilleurs peuvent accéder librement. Quant aux cueillettes en terres privées, elles sont à la discrétion des propriétaires et elles représentent une minorité des lieux où se déroulent ces pratiques.

Illustration 2 - Tenure foncière (privée et publique) du territoire au sud de la limite d'attribution de droits de coupe forestière et zones générales de cueillette

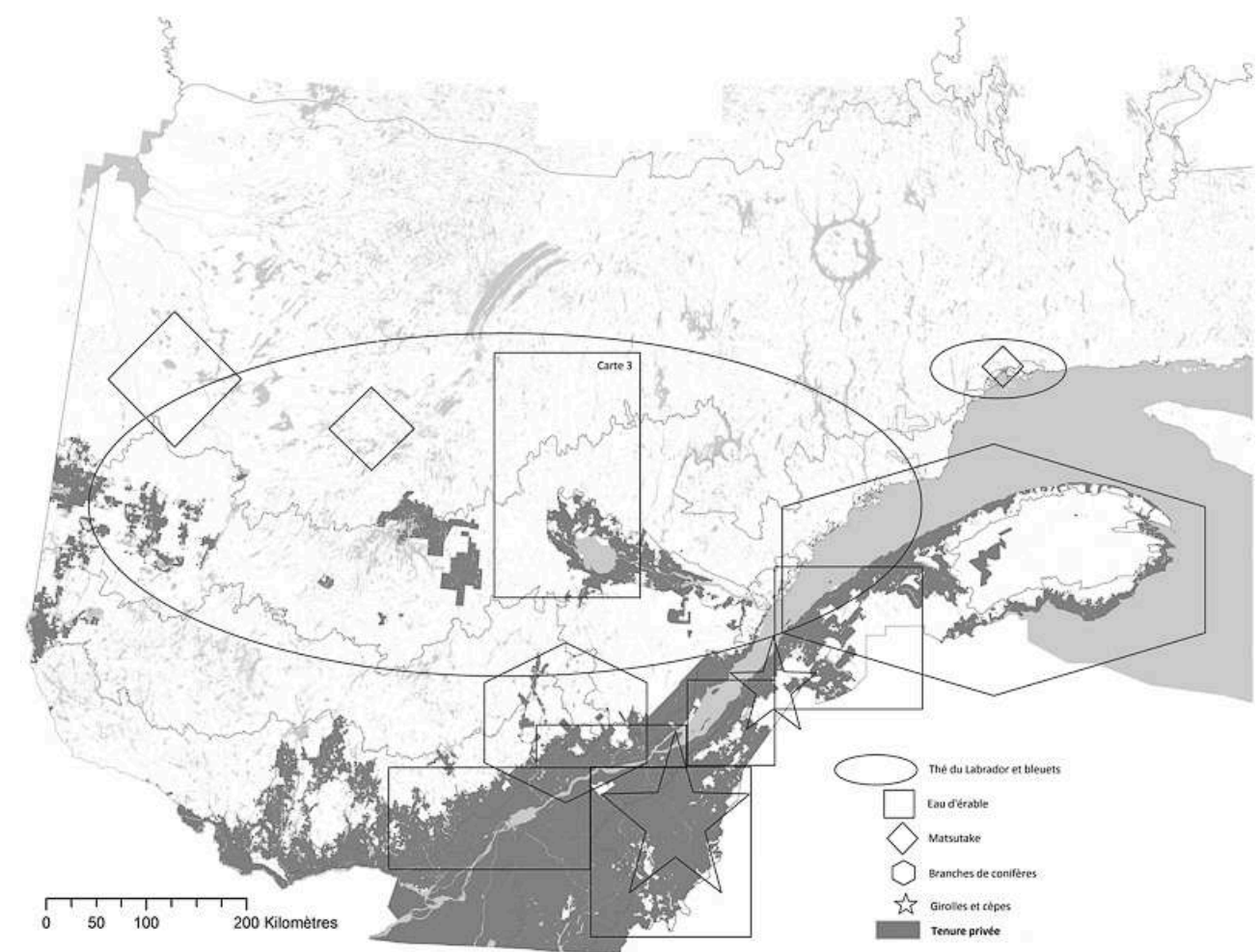

Auteur : Marc-André Brochu.

\section{Le rapport au territoire}

Les cueilleurs dont traite cet article sont des individus qui exploitent les ressources non ligneuses, principalement en terres publiques, en vue d'en faire la commercialisation. Ces cueilleurs sont généralistes, c'est-à-dire qu'ils ne sont pas spécialisés dans la cueillette d'une seule espèce ou dans l'exploitation d'un seul territoire ou type d'écosystème, bien qu'il puisse y avoir des exceptions. Uniquement au Québec, ils seraient environ 3000 individus (dont la moitié sont des cueilleurs de bleuets) se déplaçant sur tout le territoire où des cueillettes peuvent être pratiquées, et ils ne se confinent généralement pas dans un seul espace ${ }^{14}$. Leur nombre est cependant difficile à évaluer exactement, car ils pratiquent cette activité en étant souvent payés au noir, qu'il n'existe pas de réseaux formels de recrutement, de répertoire, de registre ou de données officielles les concernant. 

catégories. D'une part, ceux pour qui la cueillette est la principale activité économique rémunérée, dont des petits entrepreneurs qui développent leur entreprise en lien avec la cueillette et la transformation de PFNL et des cueilleurs autonomes à leur compte. D'autre part, il y a les cueilleurs pour qui cette activité est complémentaire et se situe dans une stratégie économique jumelant d'autres petits emplois, le chômage et le revenu minimum. Ce sont des cueilleurs qui travaillent pour ces petits entrepreneurs ou pour un gérant de camp qui approvisionne de grandes entreprises de transformation (d'huile essentielle, pharmaceutique ou de cosmétique), comme nous le verrons plus loin. Ces cueilleurs sont majoritairement des hommes d'âges variés, de la toute jeune vingtaine à la soixantaine. Certains d'entre eux sont des migrants dont le statut n'est pas régularisé. Une cueilleuse raconte : « Les cueilleurs, ce sont souvent des gens qui ont le revenu minimum ou le chômage. C'est un revenu d'appoint dans le fond. Parce que vivre de ça, un cueilleur sauvage, c'est vraiment difficile. Il faut que la personne soit très expérimentée, qu'elle connaisse déjà les lieux où sont les ressources. » La situation économique de ces cueilleurs est ordinairement précaire. engagés dans cette activité. Ils l'apprécient principalement car ils s'y sentent libres, à l'abri, tant physiquement que socialement, des tumultes urbains (et parfois même de la société), leur permettant de profiter des grands espaces. Le type de présence à la nature qu'ils sont amenés à vivre, loin de l'ambiance lourdement mécanisée de l'industrie forestière, qui est une voie souvent fréquentée pour ceux qui souhaitent vivre loin des grands centres, est aussi fort apprécié. Un cueilleur commente : « je voulais faire partie du territoire, habiter le territoire, c'est ce qui me faisait envie. C'est l'idée de travailler dans la nature sans utiliser de scie à chaîne ». Plusieurs valorisent enfin le fait d'être payés au noir et de ne " pas être dans le système ".

Les superficies arpentées par les cueilleurs varient évidemment en fonction de chaque espèce récoltée. Quelques exemples peuvent permettre d'offrir un ordre de grandeur : dans le cas du thé du labrador, un cueilleur peut parcourir un hectare par jour pendant une période de six semaines, soit environ 40-50 hectares dans une saison, au cœur de territoires situés à environ $350 \mathrm{~km}$ au nord de la ville de Québec. Les «morilles de feux ", les morilles cueillies dans les brûlis, sont cueillies à des latitudes comparables (et souvent au-delà) par des équipes de 2 à 10 personnes qui peuvent être amenées à ratisser quelques milliers d'hectares de forêts récemment incendiées au cours d'environ quatre à cinq semaines. La salicorne, quant à elle, est cueillie sur une superficie d'environ 100 hectares répartis de façon discontinue sur un mince corridor littoral de $600 \mathrm{~km}$ le long de la rive de l'estuaire et du golfe Saint-Laurent.

La durée d'une cueillette varie selon la phénologie des espèces ciblées. Le pic de densité des substances aromatiques et médicinales suit une courbe en lien avec les facteurs bioclimatiques. Certains cueilleurs suivent la vague de mûrissement des plantes et des fruits en se déplaçant en latitude (par exemple les bleuets ou les têtes de fougères). Les efforts physiques ne sont pas d'égale intensité selon la densité de couvert d'un arbuste recherché, sa fréquence de présence dans le paysage, la facilité de transport en véhicule et à pied pour y accéder et le débarder. Par exemple, pour une rémunération comparable, il est plus rapide et facile de cueillir des sommités d'arbustes de thé du labrador que de cueillir des bleuets. À poids égal, le thé rapporte plus. Aussi, il est plus simple et accessible de cueillir des branches de sapin dans des forêts de proximité qui 
permettent de revenir chez soi tous les soirs que d'aller s'établir dans un campement pour cueillir du thé du labrador.

Les territoires de cueillette peuvent être très éloignés des agglomérations humaines, par exemple à $200 \mathrm{~km}$ du village le plus proche dans le cas du thé du labrador, une distance souvent doublée pour atteindre les grands centres urbains où se trouvent les débouchés et les centres de transformation. L'accès à ces territoires est difficile, car il s'agit pour y parvenir d'emprunter des pistes des compagnies forestières qui sont souvent à l'abandon et mal entretenues. Les cueilleurs doivent être autonomes et débrouillards, en mesure de se dépêtrer de mauvaises situations si des bris mécaniques survenaient. Ces distances impliquent aussi souvent de devoir établir des campements temporaires, nous y reviendrons.

\section{L'importance des savoirs techniques et environnementaux}

Puisque les cueilleurs sont généralistes, ils doivent maîtriser différentes techniques et savoirs. La faucille en acier trempé importée de Chine est l'outil le plus communément utilisé pour la cueillette de la sommité des arbustes comme le thé du Labrador et le myrique. Dans le cas des branches d'arbres, ils ont recours à des machettes suédoises. Dans le cas des bleuets les cueilleurs utilisent ce qu'ils appellent un "peigne", un râteau fixé au-dessus d'un réceptacle qui leur permet de ratisser les buissons et de faire tomber les fruits dans le contenant sous le râteau. Plusieurs cueillettes fines, destinées notamment à des produits de niche, comme des tisanes ou des produits gastronomiques, sont faites à la main. Certaines espèces rapidement périssables, comme les quenouilles ou les champignons, nécessitent de recourir à des glacières portatives ou à une réfrigération assistée d'une génératrice.

21 La connaissance des plantes et des champignons est nécessaire à cette activité. Elle permet des cueillettes aux meilleurs moments des stades de développement des espèces afin de capter au mieux les principes actifs, les qualités aromatiques et thérapeutiques. Malgré les nouvelles offres de cours dans le domaine, la majorité des cueilleurs n'ont pas de formation officielle, collégiale ou universitaire, en biologie ou en botanique. Ils ont néanmoins constitué leur savoir par la consultation de livres de botanique, par le transfert de connaissances, généralement d'un cueilleur sénior à un nouveau cueilleur (bien qu'une forte culture du secret existe entre cueilleurs), par l'observation et par une documentation plus générale.

Des connaissances botaniques minimales sont nécessaires pour identifier et distinguer les bonnes plantes. Les cueilleurs doivent connaitre la phénologie des espèces qu'ils convoitent : les moments de la floraison et de la fructification selon les régions. Ils doivent par exemple être en mesure de prospecter fin juin, lorsqu'une plante particulière est en fleur, noter leur position et retourner les cueillir alors qu'elles sont en fruit et peu distinctes parmi les autres herbacées à la fin du mois d'août. Pour mettre en œuvre des logistiques qui s'arriment à la saisonnalité, ils doivent être mobiles et en mesure de prospecter des centaines d'hectares de terrains et de milieux écologiques divers qu'ils auront ciblés, notamment par des consultations préalables de cartes écoforestières ${ }^{15}$ et parfois de photographies aériennes (illustration 3). Les cueilleurs doivent également développer un calendrier de récolte optimisé et ajusté selon les ressources des régions qu'ils exploitent (voir tableau 1). 
Tableau 1 - Calendrier des cueillettes au Québec

\begin{tabular}{|l|l|}
\hline Mois & Types de cueillettes \\
\hline Janvier & Chaga \\
Février & Chaga, eau d'érable \\
Mars & Branches de conifère, eau d'érable \\
Avril & Bourgeons de peuplier baumier, eau d'érable \\
Mai & Prospection de sites \\
Juin & Prospection de sites, pousses \\
Juillet & Feuillage, fleurs \\
Août & Feuillage, fleurs, petits fruits, champignons \\
Septembre & Petits fruits, champignons \\
Octobre & Branches de conifère \\
Novembre & Branches de conifère \\
Décembre & Branches de conifère, chaga \\
\hline
\end{tabular}

Les cueilleurs doivent par exemple connaître et comprendre où sont les sols grossièrement sablonneux bien drainés, où se trouvent les sols organiques hydriques et comment se manifeste un écoulement latéral minéralisant. En plus de ces bases en géomorphologie et pédologie, les associations végétales dans le paysage doivent être identifiées de manière à repérer les habitats propices, notamment en reconnaissant certaines plantes compagnes. À ces connaissances, s'ajoute une sensibilité au climat, particulièrement les précipitations et le vent, des dimensions souvent subtiles, mais déterminantes au succès des efforts de récolte.

Les cueilleurs doivent savoir tirer avantage de certaines perturbations du milieu : les incendies de forêt et les coupes forestières sont des bouleversements qui dynamisent la croissance ou l'émergence d'espèces convoitées. Le thé du labrador pousse par exemple avec vigueur dans une régénération de coupe à blanc de deux ou trois ans et après le passage de certaines intensités de feux dans des milieux bien précis. Pour connaître ces lieux de coupe ou de brûlis, les cueilleurs doivent obtenir la mise à jour cartographique auprès de bureaux régionaux du ministère des Forêts, de la Faune et des Parcs.

En plus d'une planification cartographique, pour se déplacer sur le territoire, ils doivent recourir à une boussole et un appareil GPS. Les conditions de cueillettes peuvent être rudes. L'un d'eux résume ces difficultés: "c'est plutôt éprouvant, physiquement et psychologiquement. Tu es au soleil ou sous la pluie. Tu es sans cesse avec les moustiques et les taons. C'est intense. C'est pour ça que ce n'est pas tout le monde qui aime faire ça, la cueillette». Les cueilleurs doivent soutenir un effort de récolte, peu importe les conditions adverses (pluie, froidure, piqûres et morsures d'insectes). 


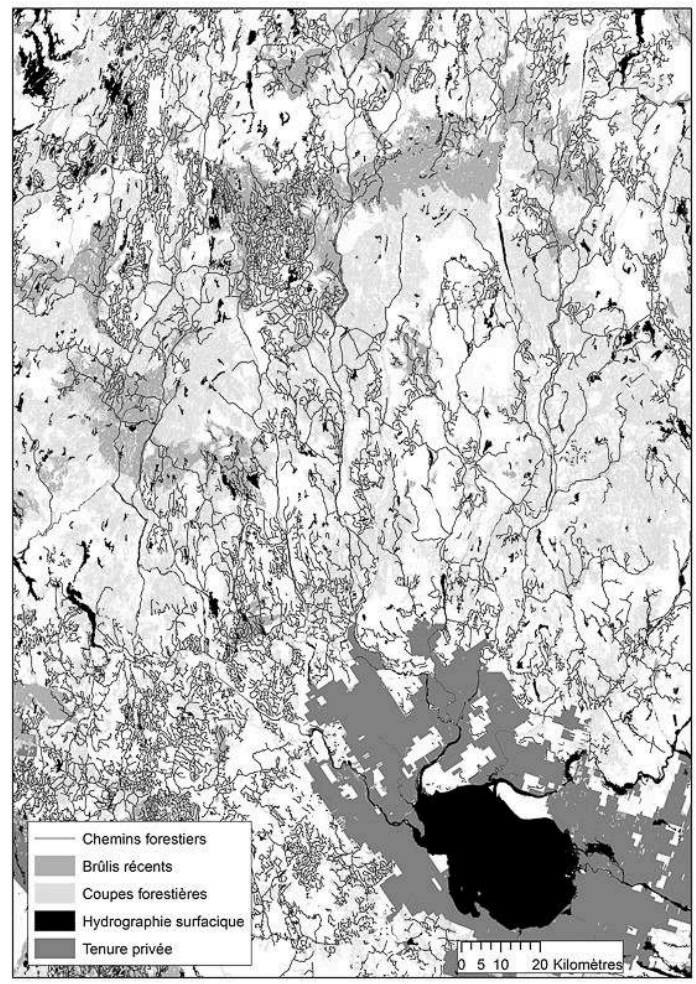

Auteur : Marc-André Brochu

\section{Organisation des activités de cueillette et de vente}

En plus de ces connaissances de base sur les espèces et les écosystèmes, les cueilleurs doivent disposer de qualités organisationnelles, de ressources et de capitaux pour se déplacer dans les territoires ainsi que pour assurer la conservation et la transformation des espèces cueillies. Plus particulièrement, les pistes, souvent mauvaises, ne sont pas adéquates pour tous les types de véhicules et nécessitent très souvent le recours à des camionnettes $4 \mathrm{X} 4$, voire à des quads. Aménagés par les industriels forestiers qui les abandonnent une fois les extractions de bois achevées, les chemins sont ultimement la propriété du gouvernement provincial qui ne dispose pas des ressources pour en assumer entièrement l'entretien (le réseau représente environ $300000 \mathrm{~km}$ ), dont celui des milliers de ponceaux et des dizaines de ponts affectés par l'érosion et les inondées de barrages de castors. Les coûts de transports pour accéder et rapporter les PFNL dans les centres de transformation, puis afin de les écouler sur les marchés des grandes villes, peuvent être prohibitifs.

\section{Les cueilleurs entrepreneurs}

27 Les cueilleurs qui ont leur propre petite entreprise de cueillette et de transformation, ainsi que ceux qui travaillent à leur compte, mais sans être formellement enregistré doivent maitriser tant les savoirs, les savoir-faire que ces ressources nécessaires à la cueillette. Ces cueilleurs peuvent ensuite vendre directement les produits frais, séchés ou transformés. Ils peuvent vendre directement au consommateur, à des restaurateurs, 
dans des marchés régionaux et en ligne. Certains cherchent à développer des produits de niche à grande valeur ajoutée. Ils peuvent aussi vendre à de plus grosses entreprises qui elles vont transformer la matière première (comme des compagnies pharmaceutiques, des compagnies de cosmétiques ou de production d'huiles essentielles). Dans le cas de produits gastronomiques, ils peuvent aussi vendre leur récolte à des courtiers, qui eux revendent à des restaurateurs. Il arrive également qu'ils vendent à des coopératives d'achat, mais ce réseau est encore en développement et en structuration et est une voie de commercialisation encore très marginale (voir tableaux 2 et 3 pour les acteurs de la cueillette et les rapports d'achat).

Ce type de cueilleur très autonome est minoritaire, mais en croissance. Il maitrise toutes les étapes du processus, de la récolte jusqu'à la vente : il planifie le calendrier des cueillettes, s'occupe de la logistique pour atteindre les sites et prélever les ressources, il est responsable de la transformation, du transport et de la gestion d'un éventuel camp temporaire. Le nombre d'entreprises ainsi conduites serait passé de moins de cinq à plus de cinquante entre 2002 et 2012 (Brodeur et al., 2012). Leurs propriétaires ont à peine plus de trente ans. Ils sont dynamiques et énergiques, compensant par leurs efforts le manque d'appui au développement de leur entreprise, qui est souvent trop petite pour bénéficier des aides des institutions financières ou des subventions gouvernementales au démarrage. Ces cueilleurs développent ces activités pour leur intérêt personnel, mais aussi dans une perspective de développement régional. C'est la façon qu'ils ont trouvé de pouvoir se trouver un emploi en région et de pouvoir y vivre, et ils ont à cœur la mise en valeur du territoire.

Tableau 2 - Acteurs de la cueillette (cueilleurs et acheteurs)

\begin{tabular}{|l|l|}
\hline Cueilleurs & Acheteurs \\
\hline Cueilleurs petits entrepreneurs & Cueilleurs petits entrepreneurs \\
(enregistrés ou incorporés) & Courtiers \\
Cueilleurs autonomes (non enregistrés & Coopératives d'achat local \\
et non incorporés) & Gérant de camp \\
Cueilleurs travailleurs & $\begin{array}{l}\text { Grande entreprises (alimentaires, pharmaceutiques, } \\
\text { cosmétiques, huiles essentielles) }\end{array}$ \\
\hline
\end{tabular}

Tableau 3 - Relations d'achat

\begin{tabular}{|l|l|l|l|l|l|l|}
\hline Acheteur & $\begin{array}{l}\text { Cueilleurs petits } \\
\text { entrepreneurs }\end{array}$ & Courtiers & $\begin{array}{l}\text { Coopératives } \\
\text { d'achat local }\end{array}$ & $\begin{array}{l}\text { Gérant } \\
\text { de } \\
\text { camp }\end{array}$ & $\begin{array}{l}\text { Grande } \\
\text { entreprise }\end{array}$ & $\begin{array}{l}\text { Marché } \\
\text { artisans et } \\
\text { internet }\end{array}$ \\
\hline $\begin{array}{l}\text { Cueilleurs petits } \\
\text { entrepreneurs }\end{array}$ & $\mathrm{x}$ & $\mathrm{x}$ & & $\mathrm{x}$ & $\mathrm{x}$ \\
\hline $\begin{array}{l}\text { Cueilleurs } \\
\text { autonomes }\end{array}$ & $\mathrm{x}$ & $\mathrm{x}$ & $\mathrm{x}$ & $\mathrm{x}$ & $\mathrm{x}$ \\
\hline
\end{tabular}




\begin{tabular}{|l|l|l|l|l|l|l|}
\hline $\begin{array}{l}\text { Cueilleurs } \\
\text { travailleurs }\end{array}$ & & & & $\mathrm{x}$ & & \\
\hline Gérant de camp & & & & & $\mathrm{x}$ & \\
\hline
\end{tabular}

\section{Les ouvriers de la cueillette}

29 La grande majorité des cueilleurs sont cependant des travailleurs, couramment au noir. Ils travaillent pour ces petites entreprises de cueillette ou pour des gérants de camps temporaires de cueillette, qui eux sont employés par de plus grandes entreprises ${ }^{16}$. Les petites entreprises emploient un nombre modeste et très ponctuel de cueilleurs, d'un à cinq, tandis que les gérants de camps au service des grandes entreprises peuvent solliciter les services d'une centaine de cueilleurs pendant une saison. Ces cueilleurs ne sont pas, pour la majorité, embauchés de façon stable et récurrente. Ils ne sont pas fidèles à une entreprise ou à un gérant plutôt qu'un autre ; ils choisissent, au gré des saisons et des prix offerts pour leur service, vers qui ils se tourneront. Il arrive aussi que d'aucuns choisissent le temps d'une saison de ne pas se consacrer à la cueillette au profit d'une autre activité, ou d'aller plutôt cueillir ailleurs, l'Ouest canadien étant une destination privilégiée en ce sens.

Le gérant d'un camp de cueillette a comme mandat d'approvisionner l'entreprise et a carte blanche pour y parvenir. C'est lui qui doit trouver la ressource sur le territoire. L'entreprise qui l'engage (pharmaceutique, cosmétique, alimentaire, huile essentielle) ne se mêle d'aucun des aspects liés à la cueillette ou aux cueilleurs, et elle ne cherche qu'un produit cueilli avec des qualités déterminées. Le gérant de cueillette coordonne les activités et le travail des cueilleurs, il contrôle le calendrier et les sites de cueillettes, les campements temporaires, ainsi que le savoir sur les espèces et les territoires. Il coordonne les cueilleurs en leur donnant des spécifications quant aux espèces à cueillir et à la manière de procéder pour les récolter et les transformer. Il doit assurer une constance de l'approvisionnement, tant en quantité qu'en qualité, tout au long de la saison et il est responsable du transport des récoltes vers les lieux de transformation, généralement à l'aide d'un camion semi-remorque. C'est lui qui est payé par la grande entreprise, selon le poids apporté. Les cueilleurs sont quant à eux payés par le gérant en argent au poids. De ce montant sont déduits les coûts de logement et de nourriture ${ }^{17}$. Le camp de travail temporaire compte en général une vingtaine de cueilleurs qui sont hébergés dans des roulottes ou dans des tentes. Une des tentes abrite la cuisine du campement et une autre la pesée. Dans le cas du thé du labrador, un cueilleur peut rapporter en une journée de travail environ $200 \mathrm{~kg}$ de ramures. Pour ce faire, il commence en général sa journée vers 3 heures, préférant profiter de la fraîcheur du matin ${ }^{18}$.

31 Puisque les cueillettes varient chaque année, ainsi que la demande et le nombre de cueilleurs, que les quantités prélevées ne sont pas recensées par un organisme qui systématise ces informations à l'échelle du Québec ou du Canada et que les prix des espèces ne sont pas réglementés par un marché ou par le gouvernement, il nous est impossible de connaître exactement les prix offerts et les revenus des cueilleurs. Il nous est possible cependant d'estimer que ces cueilleurs peuvent globalement gagner entre 200 et $500 \$$ par jour selon l'abondance et le type d'espèce cueillie. Par exemple, un 
cueilleur de sommités d'arbustes de thé du labrador peut obtenir $1,85 \$$ le $\mathrm{kg}$, soit environ 10000 à $15000 \$$ au cours d'une période d'activité de cinq à six semaines. Les glaneurs de branches de conifère (sapin, pruche, pins, épinette noire) reçoivent 0,18 \$ le $\mathrm{kg}$ une fois découpé à la machette (gros bout maximal de $3 \mathrm{~cm}$ ) et mis en fagot de $30 \mathrm{~kg}$.

La main-d'œuvre est volatile et les entreprises qui en dépendent peuvent en pâtir. Ces entreprises affirment vivre une pénurie de travailleurs et constatent parallèlement leur vieillissement (Brodeur et al., 2012). Les entreprises et les gérants de camps doivent s'assurer la disponibilité d'un nombre suffisant de cueilleurs pour les périodes d'abondance limitées dans le temps, comme pour les bleuets ou le thé du labrador. Dans ces périodes, les cueilleurs peuvent bénéficier de la forte demande pour exiger de meilleurs prix pour leurs récoltes. La même situation survient lorsque des périodes de cueillettes se chevauchent et que les prix varient entre les espèces, par exemple entre les morilles et les branches de sapin.

\section{Développement régional et surexploitation des ressources}

Enfin, certains cueilleurs pratiquent cette activité en tant que membres d'une coopérative forestière. Dans ces cas, la plupart de ces cueilleurs sont des travailleurs forestiers chargés de travaux de débroussaillage et de dégagement. Ils peuvent se consacrer à la cueillette au cours de périodes d'abondance de certains champignons ou lors de périodes de ruptures des activités de l'industrie ligneuse. Comme pour les petits entrepreneurs, ce modèle d'organisation de la production s'inscrit dans une perspective de développement régional, comme cela a été le cas dans la région du Saguenay. Ces initiatives permettent notamment de diversifier les sources de revenus des coopératives forestières, car les coupes de bois sont à la baisse depuis 15 ans et les perspectives d'avenir sont sombres, et d'assurer le maintien d'emplois locaux pour leurs habitants, contribuant à la vitalité globale de la région.

La cueillette est une activité individuelle et les cueilleurs sont en compétition entre eux pour accéder les premiers aux meilleurs sites de cueillette et pour en obtenir les meilleurs volumes. Le premier arrivé est le premier servi. Avec la popularité croissante de cette activité, certains cueilleurs plus âgés qui avaient leurs habitudes et leur «territoire informel » de cueillette les voient pillés par de nouveaux venus dans un climat de far west (Brodeur et al., 2012). Certains cueilleurs craignent que des espèces soient mal exploitées ou surexploitées. Les méthodes de cueillette apparemment artisanales (par exemple le recours à la faucille) peuvent notamment avoir un impact sur la vigueur de la ressource lorsqu'appliquées par des dizaines de cueilleurs dans un même secteur d'intervention. Une cueilleuse le souligne concernant le cas plus précis de la fougère tête de violon : « C'est dangereux de trop la cueillir, puis de la tuer. C'est une plante qui est très vulnérable. Puis les gens, souvent, ils ne savent même pas ça. Ils arrivent dans une place, puis elle a déjà été cueillie, puis ils cueillent encore. Puis l'autre revient, ça repousse, il recueille encore. Bien, la plante, elle va mourir dans deux, trois ans [...]. C'est une plante qui peut vivre des centaines d'années si tu sais comment la cueillir. Il faudrait vraiment que ça soit contrôlé ». Le moment de certaines coupes peut contribuer à épuiser les ressources, comme lorsque le pic aromatique de certaines plantes chevauche une des phases critiques de leur cycle de vie, tel l'aoûtement. 

énoncée par les petits entrepreneurs, qui la situent plus particulièrement dans une perspective de développement local et de retombées régionales de l'exploitation du territoire : «Je trouve que la cueillette, ça devient bien à la mode, et j'ai la crainte que ce soit un peu n'importe qui qui marche là-dedans. Pas nécessairement du monde passionné. Plutôt du monde de l'extérieur qui vient accaparer quelque chose qui aurait pu être un moteur local. En tout cas, d'un point de vue de la gestion de la ressource, disons qu'il y a quelque chose à faire. » Argumentant la nécessité de contrôle, certaines entreprises demandent au gouvernement que soient développées des concessions qui délimiteraient des territoires où l'exploitation de telle ou telle espèce leur serait exclusive. Certaines terres leur seraient alors réservées sans qu'elles aient à en être propriétaires, selon le modèle de gestion de la ressource ligneuse dans les forêts publiques. Cela soulève cependant des questions, comme l'expose cette femme propriétaire d'une petite entreprise de cueillette et elle-même cueilleuse: "Mais, éventuellement, il va y avoir des permis de récolte qui vont être émis. Mais, alors, après ça, qui ça va acheter les permis de récolte ? Est-ce que ce sera quelqu'un de riche qui va... qui va acheter tous les permis de récolte? Comme pour les quotas de pêche?» Cette approche ne fait pas l'unanimité parmi les entrepreneurs et les cueilleurs.

\section{Capter la valeur en la cueillant : diversité socio- environnementale de la cueillette}

La cueillette commerciale est une activité en croissance au Québec depuis quelques décennies. En raison du contexte politique et économique de la province, la structuration de ces activités y est relativement récente. L'intérêt économique qu'elle peut offrir se confirme, mais il voile la diversité des pratiques de cueillette et la fluidité de l'organisation qu'elle suppose et qui sont au cœur du succès de ces initiatives. Ces caractéristiques se déploient dans un "espace péricapitaliste " où se côtoient et s'amalgament des processus qui sont à la fois marginaux et partie prenante du capitalisme.

La cueillette nécessite un savoir important et généraliste du territoire, de ses pistes, des plantes, des écosystèmes, des saisons. Elle implique aussi la maîtrise de compétences, de ressources et la possession d'un capital pour accéder aux espèces sauvages, les transformer et les transporter. Les cueilleurs peuvent parcourir le territoire sans contraintes d'accès liés à la tenure. Ces espaces sont habituellement éloignés, parfois austères, souvent vidés de leurs ressources ligneuses par le passage précédent de l'industrie forestière. Le prélèvement des espèces s'y fait à l'abri des regards, pouvant potentiellement mener à des abus délétères. Les cueilleurs s'intéressent à une nature dont les caractéristiques ne sont pas aisément contrôlables, manipulables, ils doivent tenir compte de ses cycles et de l'hétérogénéité des distributions et des abondances ne se laissant pas facilement approprier et enchâsser dans une logique de chaîne d'approvisionnement aux paramètres fixes. L'organisation de leur travail est souple, sans recrutement formel, sans formation obligatoire ou disciplinaire, sans processus d'embauche encadré, sans composition d'équipes préalables ou d'horaires fixes. Ces caractéristiques particulières du travail de la cueillette se sont avérées essentielles à la pratique de cette activité et contribuent à son déploiement capitaliste.

EchoGéo, 47 | 2019 

coûts socio-environnementaux. Les entreprises qui achètent et transforment les récoltes captent les matières premières grâce à une force de travail qui leur est invisible, sans avoir à contrôler les conditions de leur production, sans avoir à investir dans la reproduction et la mise en croissance des divers PFNL et elles bénéficient de leur capacité même de se régénérer sans entretien dans des sites abandonnés par d'autres industries d'exploitation en ayant un accès libre à des territoires publics. Les caractéristiques de l'organisation du travail permettent aux entreprises de capter le savoir et le savoir-faire des cueilleurs, qui sont souvent des travailleurs "fantômes ", à qui elles n'ont pas à payer d'avantages sociaux et face à qui elles n'ont pas de responsabilités légales.

Cette accumulation par captation se déployant dans un espace péricapitaliste contribue aux inégalités sociales et économiques et contribue à un processus de différenciation en cours propice à une exploitation des travailleurs et une surexploitation de la nature. Les savoirs et les ressources nécessaires à la cueillette ne sont pas homogènes et uniformément répartis entre les cueilleurs, les petits propriétaires et les gérants étant généralement mieux pourvus que les travailleurs, plus précaires, marquant une distinction importante qui maintient une différentiation socio-économique à laquelle s'ajoutent souvent des situations de marginalité politique et économique pour ces derniers. Les petites entreprises régionales sont pour leur part plus précaires que les grandes en ce qui concerne les capacités de transformation et de vente, marquant une différentiation économique accentuée par la disparité entre le développement des régions et des grands centres.

Pour contrer ce processus de différenciation, les petites entreprises régionales tentent notamment de proposer des modèles de structuration organisationnelle, par des activités de formation et de professionnalisation et un encadrement des cueilleurs, mais sans toutefois parvenir encore à rejoindre une masse critique conséquente d'acteurs. Elles sont préoccupées par la protection des ressources et les retombées régionales de l'extraction de la nature de leur territoire. Elles cherchent à ajouter une profondeur à cet intérêt économique en visant la mise en œuvre d'une manière d'habiter le territoire, de pouvoir bien vivre en région par le développement local. Elles cherchent en quelque sorte à développer une collaboration (Tsing, 2012) entre les espèces cueillies, les habitants locaux et les réseaux socio-économiques et en ce sens à transformer le modèle de captation des valeurs en cours par de nouveaux partenariats socio-environnementaux.

\section{Conclusion}

Les activités de cueillette approvisionnent un marché global et industriel dont les demandes, tant pour les secteurs alimentaire, aromatique que pharmaceutique, sont en croissance et le Québec n'y échappe pas. Elles s'y déploient grâce à la mise en œuvre par différents cueilleurs de pratiques diversifiées et dont l'organisation est flexible, suivant les caractéristiques qu'imposent les espèces elles-mêmes dans des espaces " péricapitalistes ». La diversité socio-environnementale, la nature et l'organisation des cueillettes, jumelant des activités relevant du capitalisme et d'autres évoluant à sa marge, est ce qui a permis le foisonnement de ces pratiques.

EchoGéo, 47 | 2019 

différente de ces pratiques par leur structuration et leur formalisation, avec pour objectif de favoriser le développement régional et l'économie locale des régions où sont réalisées les cueillettes. Ces formalisations auront-elles un impact sur une activité qui a réussi à se développer notamment grâce à ses caractéristiques informelles? Les variabilités écologiques et territoriales le permettent-elles, car il s'avère généralement exigeant et non sans conséquence de discipliner la nature (Scott, 1998) ? Les cueilleurs québécois le souhaitent-ils? Ce n'est pas toujours le cas ailleurs, les cueilleurs de l'Orégon que présente Tsing (2015:77) ne semblent pas pour leur part favorables à cette structuration formelle. Ces formalisations auront-elles pour conséquences de renforcer la marginalisation des régions et leurs efforts de dynamisation socioenvironnementale? A contrario, quelles seraient les implications de ne pas le faire? Ces initiatives récentes peuvent-elles jeter un nouvel éclairage sur les possibilités que peuvent offrir les espaces péricapitalistes pour penser le monde autrement?

\section{BIBLIOGRAPHIE}

Abanda F., Boucher J. L., Bouthillier L., Chiasson G., 2016. Du village forestier à la gouvernance locale : la persistance de l'aspiration à la participation. Revue vie économique, vol. 8, nº 1, p. 1-8. Biopterre, 2015. Diagnostic et étude d'opportunités de la filière des produits forestiers non ligneux. Rapport final. Sainte-Anne-de-la-Pocatière, Biopterre - Centre de développement des bioproduits, $47 \mathrm{p}$.

Biopterre, 2016. Journée de réflexion sur l'essor de la filière PFNL du Bas-Saint-Laurent - 30 mai 2016. Compte rendu. Sainte-Anne-de-la-Pocatière, Biopterre - Centre de développement des bioproduits, $26 \mathrm{p}$.

Bouthillier L., Hanin F., Lapointe P.-A., 2011. Orientations relatives à la sélection, à la mise en place et au fonctionnement des forêts de proximité. Mémoire déposé au Ministère des ressources naturelles et de la faune du Québec dans le cadre de la consultation publique sur la forêt, $56 \mathrm{p}$.

Brodeur C., Gilbert D., Dostie S., Bernier P., Gilbert A.-M., 2012. Planification stratégie du secteur des PFNL au Saguenay-Lac-St-Jean. Rapport complet. Québec, Groupe AGÉCO, 83 p.

Charron I., Dostie S., St-Arnaud R.-M., 2014. Plan de développement stratégique pour l'industrie des produits forestiers non ligneux du Québec. Rapport final. Québec, Groupe AGÉCO, 23 p.

Fortin J.-C., Lechasseur A., 1993. Histoire du Bas-Saint-Laurent. Québec, Bibliothèque nationale du Québec, 860 p.

Fugère A., Léveillé C.-A., 2005. Produits forestiers non ligneux en Gaspésie : Inventaire des ressources humaines et des infrastructures. Aperçu de la situation actuelle de la mise en marché. New Richmond, Fédération des groupements forestiers de la Gaspésie et Le Syndicat des producteurs de bois de la Gaspésie, 211 p.

Gagnon S., 2009. L'appropriation ludique de la forêt au Québec : d'une gestion privée de « club » à une intervention publique de protection (1885-1935). Études caribéennes, n 12, p. 1-17.

EchoGéo, 47 | 2019 
Gélinas N., Bernard A., Marcotte P., Latorre J., 2016. Mycotourisme : pratique touristique pour un développement socio-économique durable et viable en régions forestières. Québec, Institut EDS, $32 \mathrm{p}$.

Jean B., 1989. Le développement régional à l'heure du développement local : le temps des incertitudes. Canadian Journal of Regional Sciences/Revue canadienne des sciences régionales, vol. 12, $\mathrm{n}^{\circ} 1$, p. 9-24.

Lapointe P.-L., 2010. La chasse et la pêche : des loisirs enracinés dans l'univers des Québécois. BANQ, n 82, p. 2-3.

Ministère des Ressources naturelles et de la Faune (MRNF), 2003. Aperçu des produits forestiers non ligneux (PFNL). [En ligne]. http://www.mrnf.gouv.qc.ca/publications/forets/entreprises/ apercu_PFNL.pdf

Ministère des Ressources naturelles et de la Faune (MRNF), 2009. Ressources et industries forestières. Portrait statistique. [En ligne]. http://www.mrnf.gouv.qc.ca/publications/forets/ connaissances/stat_edition_complete/complete. pdf

Mitchell D. A., Tedder S., Brigham T., Cocksedge W., Hobby T., 2010. Policy gaps and Invisible Elbows : NTFPs in British Columbia. In Laird S. A., McLain R. J., Wynberg R. P., Wild Products Governance : Finding Policies that Work for Non-Timber Forest-Products. London, Earthscan, p. 113-34.

Murphy B., Chrétien A., Morin G., 2017. Maple syrup value system and value chains : considering indigenous and non indigenous perspectives. In Bullock R., Broad G., Palmer L. Smith P., Growing community forests, practice, research and advocacy in Canada. Winnipeg, University of Manitoba press, p. 186-210.

Scott J. C., 1998. Seeing Like a State. How Certain Schemes to Improve the Human Condition Have Failed. New Haven, London, Yale University Press, 460 p.

Turgeon M., 2003. Aperçu des produits forestiers non ligneux (Pfnl). Québec, Gouvernement du Québec, MRNFP, $15 \mathrm{p}$.

Tsing A., 2012. Unruly Edges : Mushrooms as Companion Species. Environmental Humanities, vol. 1, $n^{\circ} 1$, p. $141-54$

Tsing A., 2015. The Mushroom at the End of the World. Princeton, Oxford, Princeton University Press, $352 \mathrm{p}$.

\section{NOTES}

1. L'idéation des PFNL est plus ancienne ailleurs dans le monde, notamment en Afrique, impulsée par la FAO pour soutenir et diversifier les économies locales (FAO 2001, 2007).

2. Ils sont considérés comme des PFNL car ils ne sont pas exploités pour leur valeur de transformation ligneuse (matériaux de construction, pâtes et papier, chauffe).

3. La recherche a été subventionnée par le Conseil de recherche en science humaines du Canada (Conservation environnementale et patrimonialisation de la nature 2013-2019) et elle a impliqué la participation, sous la direction de Sabrina Doyon, de deux professeurs-chercheurs, de trois étudiants de maîtrise et de deux de doctorat.

4. Les autochtones sont des acteurs primordiaux en ce qui concerne la cueillette de plantes sauvages, mais nous ne les aborderons pas ici.

5. Après la conquête des Anglais (1759-1760), ces derniers prirent les rênes de l'administration d'État et des ressources naturelles du Bas-Canada, qui devint le Québec, en évinçant les Canadiens-français. Les "Barons du bois", de riches familles anglaises, ont bénéficié de ce 
système et ont pu s'enrichir de leurs activités, alors que les Québécois étaient employés comme bûcherons dans les concessions. Les luttes autonomistes puis souverainistes de la deuxième moitié du $20^{\mathrm{e}}$ siècle ont cherché à briser le système de privilèges qui favorisait jusqu'alors les descendants d'Anglais. L'accession aux terres publiques concédées et le démantèlement des clubs privés s'inscrivent dans cette foulée.

6. Voir aussi les travaux de B. Jean sur le développement régional au Québec et les rôles qu'y jouent l'emploi et l'occupation du territoire (1989).

7. Épilobe Chamaenerion angustifolium, rosier Rosa rubiginosa, achillée Achillea millefolium, millepertuis Hypericum perforatum, marguerite Leucanthemum vulgare, plantain Plantago rugelii, asclépiade Asclepias syriaca, chicoutée Vaccinium oxycoccos, verge d'or Solidago puberula, comptonie voyageuse Comptonia peregrina, bleuet Vaccinium angustifolium, thé $\mathrm{du}$ labrador Rhododendron groenlandicum, etc.

8. Livèche Ligusticum scoticum, fougère à tête de violon Matteuccia struthiopteris, canneberge Vaccinium oxycoccos, quenouille Typha angustifolia, salicorne Salicornia depressa, myrique Myrica gale, sagittaire Sagittaria latifolia, etc.

9. Thé des bois Gaultheria procumbens, actée Actaea rubra, dentaire carcajou Cardamine diphylla, sanguinaire Sanguinaria canadensis, etc.

10. Sapin Abies balsamea, thuya Thuja occidentalis, if Taxus canadensis, et thé du labrador (forme où les sommités sont coupées, et non seulement les feuilles).

11. Bouleau à papier Betula papyrifera, érable à sucre et rouge Acer saccharum, Acer rubrum.

12. Bolets (Hemileccinum subglabripes, Leccinum piceinum Boletus chippewaensis), morilles (Morchella elata, Morchella americana), chanterelles (Cantharellus roseocanus Craterellus tubaeformis), trompettes de la mort (Craerellus fallax), dermatose des russules (Hypomyces lactifluorum mycoparasite sur basidiomes de Russula brevipes), matsutake (Tricholoma magnivelare), chaga (Inonotus obliquus), etc.

13. Picea mariana, Tsuga canadensis, Betula alleghaniensis.

14. Pour des raisons de convenance (proximité des lieux d'habitation, étendue du territoire de cueillette importante, barrière de la langue), ils demeurent pour la majorité dans les limites de la province de Québec.

15. Les cartes écoforestières sont maintenant libres d'accès sur l'Internet, leur pleine utilisation demande cependant des compétences de géomatique et des techniques forestières. Les cartes permettent d'obtenir des informations sur les peuplements, les types de couvert, l'âge des arbres, les compositions en essence, la géomorphologie des sols (dépôts, textures, drainages). Ces cartes étaient auparavant en vente en feuillets de $1: 20000$

16. Ce sont des entreprises de fabrication d'huiles essentielles, de produits pharmaceutiques, cosmétiques ou alimentaires qui ont des chiffres d'affaire de plus d'un million de dollars annuellement. Nous n'explorons pas leurs filières de marchandisation ici.

17. Dans les dernières années, des entreprises ont recruté des travailleurs saisonniers migrants, embauchés dans le cadre du programme canadien de travailleurs migrants. Leur rétribution et les conditions de leur embauche varient dans ces cas. Nous n'abordons pas ces cas dans cet article.

18. Les relations entre ces personnes ne peuvent être abordées dans cet article pour des contraintes d'espace. 


\section{RÉSUMÉS}

La cueillette commerciale d'espèces sauvages est une activité dont la popularité est en croissance au Québec. Si les produits cueillis sont de mieux en mieux connus, on ne peut en dire autant de l'activité elle-même et des cueilleurs. Qui sont-ils ? Comment accèdent-ils aux ressources ? Quels défis rencontrent-ils? Cet article aborde ces questions par le truchement de la diversité des pratiques déployées. Les notions «d'accumulation par captation» et «d'espaces péricapitalistes", permettant d'explorer les différentes configurations socio-environnementales et économiques des activités de cueillette, guident l'exploration. Pour ce faire, l'article brossera un portrait du développement des activités de cueillette au Québec et présentera des caractéristiques des cueilleurs, de leurs savoirs, de leurs pratiques, des territoires qu'ils explorent et de l'organisation de leur travail.

Commercial wild plant picking is an increasingly popular activity in Québec. While those products are now better known, the activity and the pickers themselves are not. Who are they? How do they get access to the natural resources? Which challenges are they facing? This paper tackles these questions exploring the various practices put forward by pickers. Tsing's concepts of "salvage accumulation" and "pericapitalist spaces" guide this study, which analyzes the development of picking activities in Québec and presents some of the pickers main characteristics, their local knowledge, practices, territories, and work organization.

\section{INDEX}

Mots-clés : cueillette, Québec, produit forestier non-ligneux PFNL, anthropologie Keywords : wild plant picking, Québec, non timber wood product NTWP, anthropology

Thèmes : Sur le Champ - Sur le Terrain

\section{AUTEUR}

\section{SABRINA DOYON}

Sabrina Doyon, sabrina.doyon@ant.ulaval.ca, est Professeure au département d'anthropologie, Université Laval et Présidente de la Société canadienne d'anthropologie. Ses dernières publications :

- Doyon S. (dir), 2019 (sous presses), D'espoir et d'environnement? Nouvelles ruralités, activités alternatives et mise en valeur de la nature au Bas-St-Laurent (Québec). Québec, Presses de l'Université Laval.

- Doyon, S., 2019 (sous presses), Conservation environnementale et production alimentaire « alternative » au Bas-St-Laurent, Québec. Anthropologie et sociétés, vol. 43, n³.

- Doyon, S. 2018. Pêches des bélugas, des anguilles et des esturgeons noirs de l'estuaire du SaintLaurent : entre la protection et la marchandisation. Ethnoécologie [En ligne], n 13. http:// journals.openedition.org/ethnoecologie/3472 